\title{
The Relationship between MMP-9 and Infarct Related Artery Reflow in Acute STEMI Patients
}

\author{
Fangming Guo ${ }^{1^{*}}$, Weili Chai ${ }^{2 \#}$, Meipin Liu ${ }^{1}$, Chunbao $\mathrm{Yu}^{3}$, Wenbo Liu ${ }^{1}$ and Xiaohuan Wang ${ }^{4^{*}}$ \\ ${ }^{1}$ Department of Cardiology, YantaiShan Hospital, Yantai-264001, China \\ ${ }^{2}$ Department of Cardiology, Third People's Hospital of Jinan City, Jinan-250101, China \\ ${ }^{3}$ Katharine HSU International Research Center of Human Infectious Diseases, Shandong Chest Hospital, Jinan 250013, China \\ ${ }^{4}$ Department of Cardiology, Gansu Provincial Hospital, Lanzhou City-730000, China \\ \#Fangming Guo and WeiLi Chai contributed equally to this study
}

"Corresponding authors: Fangming Guo, Department of Cardiology, YantaiShan Hospital, No.91 Jiefang Road, Yantai 264001, Shandong, P.R. China, Tel: 0865356602193; Fax: 086-535-6602108; E-mail: guofmcn@qq.com; Xiaohuan Wang, Department of Cardiology, Gansu Provincial Hospital, No. 204, Donggang West Road, Lanzhou City, 730000, P.R. China, Tel: 08613669322973; E-mail: 15953550208@qq.com

Received date: June 25, 2017; Accepted date: July 10, 2017; Published date: July 15, 2017

Copyright: ( 2017 Guo F, et al. This is an open-access article distributed under the terms of the Creative Commons Attribution License, which permits unrestricted use, distribution, and reproduction in any medium, provided the original author and source are credited.

\begin{abstract}
Objective: Atherosclerotic plaque rupture leading to coronary artery occlusion is the culprit event which underpins a majority of acute myocardial infarction, and Matrix metalloproteinases (MMPs) contribute to atherosclerotic plaque rupture by involving in extracellular matrix degradation and artificial balloon extrusion. Patients with ST-segment elevation myocardial infarction (STEMI) caused by plaque rupture are at high risk for slow reflow, but the relationship between reflow and MMP-9 remains unclear, especially in the real world of local plaque rupture. We investigated the association between the levels of matrix metalloproteinase-9 (MMP-9) in infarct-related arterial infusion and the risk of slow reflow in STEMI patients following percutaneous coronary intervention $(\mathrm{PCl})$.

Methods: 65 eligible acute STEMI patients undergoing successful $\mathrm{PCl}$ were included in the current study. Blood samples were obtained from the extraction catheter placed distal to the lesion during PCI. Plasma MMP-9 levels were determined by immunoassay method.

Results: Using multiple logistic regression analysis, MMP-9 levels (OR 0.881, $\mathrm{Cl} 0.791-0.981 ; \mathrm{P}=0.021$ ) was found to be a significant risk factor of slow flow together with HSCORE (OR=0.085, Cl 0.014-0.506; $\mathrm{P}=0.007)$. ROC curve with area under the curve $(0.740)$ and $95 \%$ confidence interval $(0.607-0.872)$ revealed that lesion MMP-9 changes had an predictive value for no reflow( $\mathrm{P}=0.002)$.

Conclusions: The present study indicated that plasma MMP-9 levels in the culprit coronary artery was associated with slow flow in patients with ST-elevation MI following successful primary PCI.
\end{abstract}

Keywords: Matrix metalloproteinase; Coronary artery reflow; Atherosclerotic plaque; Percutaneous coronary intervention

\section{Introduction}

Atherosclerotic plaque rupture is the culprit event which underpins a majority of acute myocardial infarction (AMI). Matrix metalloproteinases (MMPs) contribute to atherosclerotic lesion progression by involving in extracellular matrix degradation [1,2]. With the progress of disease, the over-expression of these enzymes lead to thinning of the plaque fibrous cap, resulting in plaque instability, rupture and thrombosis and thus development to acute ischemic in the clinical [3].

Atherosclerosis of all cells in the vessel wall can secrete several metalloproteinase [4], but MMP-9 was secreted mainly by the inflammatory cells [5,6]. In atherosclerotic plaque of AMI, MMP-9 is more concentrated in the local of plaque which has more foam cells [7], early elevated of the level of MMP-9 in plasma in the patients with acute coronary syndrome has relationship with the activity of unstable plaque [8]. Patients with STEMI caused by plaque rupture are at high risk for slow reflow, but the relationship between reflow and MMP-9 remains unclear, especially in the real world of plaque rupture. In this study we investigated the possible relationship between the elevated of the level of MMP-9 in the lesion or peripheral in plaque of acute myocardial infarction with slow blood flow after percutaneous coronary intervention (PCI).

\section{Materials and Methods}

\section{Patient population}

We consecutively recruited 70 eligible STEMI patients, who received successfully primary percutaneous coronary intervention as needed in Yantai Hill Hospital. Inclusion criteria were prolonged chest pain $(>30$ $\mathrm{min}$ ) no responding to nitroglycerin infusion, ST-segment elevation $\geq$ $0.2 \mathrm{mV}$ in two or more adjacent leads on standard electrocardiogram, more than double the upper normal limits of creatine kinase (CK), $\mathrm{CK}-\mathrm{MB}$, or relative index, and successful primary PCI performed within $12 \mathrm{~h}$ of the onset of chest pain. Stent implantation was successfully performed in all patients.

No significant side-branch occlusion occurred during the procedure. Overall, 5 patients were excluded from the study, due to time from symptom onset $\geq 12 \mathrm{~h}(\mathrm{n}=4)$ or missing of blood sample for MMP-9 measurement $(n=1)$. Thus, 65 patients (age $62.5 \pm 10.5$ years, 
38 males, mean $1.54 \pm 0.53$ stent was used per patient) were eventually included in the study. This study was conducted in accordance with the declaration of Helsinki. This study was conducted with approval from the Ethics Committee of Yantai Hill Hospital. Written informed consent was obtained from all participants.

\section{Primary PCI}

All patients included in the study were given $300 \mathrm{mg}$ oral aspirin, $300 \mathrm{mg}$ clopidogrel in the emergency room. The patients were transferred to the cardiac catheterization laboratory and primary PCI was then performed. PCI procedures were performed through radial or femoral $(n=3)$ approach with a 6 French guiding catheter. A bolus of $5000 \mathrm{IU}$ of heparin was administered. Selective coronary angiography was performed after the intracoronary administration of nitroglycerin, at least five standardized views of the left coronary artery and three views of the right coronary artery were obtained. After conventional wire crossing, Diver CE (Innovative technologies, Italy) was used in all STEMI patients only if a total occlusion (thrombolysis in myocardial infarction [TIMI] flow 0) existed, then direct stenting implantation was performed whenever possible, preceded by balloon predilatation if necessary.

\section{Blood sampling protocol and determinations}

Blood samples were drawn from a brachial vein in all patients at the time of admission. Blood was collected in EDTA tubes or tubes without any anticoagulan and centrifuged. Plasma and serum aliquots were stored at -80 in appropriate cuvettes until assayed. C-reactive protein serum levels were measured using an immune nephelometric high-sensitivity method (DADE Behring, Milan, Italy). Serum cardiac enzymes (creatine kinase $[\mathrm{CK}]$ and CK-MB fraction) were measured every $4 \mathrm{~h}$ during the first day and every $24 \mathrm{~h}$ in the following 3 days using standardized methods.

To detect MMP-9 at the lesion site during PCI, Diver CE, a 6-F compatible rapid exchange clot extraction catheter, was advanced along the guidewire and positioned $10-20 \mathrm{~mm}$ distal to the coronary stenosis. Sets of blood samples were drawn from the lesion by extraction catheter before balloon angioplasty. All blood samples were drawn slowly in all cases to minimize factors activation. All samples were immediately put on ice and centrifuged at 3,500 rpm for $10 \mathrm{~min}$ at $4^{\circ} \mathrm{C}$, then frozen at $-80^{\circ} \mathrm{C}$ until the assays were performed. Levels of serum MMP-9 was determined by ELISA according to the manufacturer's instructions.

\section{Assessment of flow}

TIMI flow was assessed according to previous studies [9]. TIMI flow grade was assessed at an angiographic core laboratory as previously defined [1]. The CTFC is the number of cine frames required for contrast to first reach standardized distal coronary landmarks in the culprit artery and is measured with a frame counter on a cine viewer. The CTFC is a measure of time and data were converted when necessary to be based on the most common filming speed used in the United States (30 frames/s).

The CTFC was divided by 30 to calculate the transit time for dye to traverse the length of the artery to the landmark in seconds and multiplied by 1000 to calculate the time in milliseconds. This was used along with the heart rate to calculate the fraction of a cardiac cycle required for dye to traverse the artery: fraction of cardiac cycle $=($ CTFC $/ 30$ seconds $) /(60$ seconds/heart rate $)$. Calculation of the fraction of a cardiac cycle required for dye to traverse the culprit artery normalizes the CTFC for heart rate. MBG was assessed according to van't Hof et al. criteria [10]. We defined angiographic no-reflow as a coronary TIMI flow grade $\leq 2$ after vessel reopening or TIMI flow 3 with a final $M B G \leq 2$ (modified from Gibson et al.) [11].

Collateral grading was done according to the Rentrop grading system that ranges from 0 (no collateral filling) to 3 (complete vessel opacification by retrograde flow) [12]. Thrombus score was modified from Gibson et al. [11]. Angiographic assessment was always performed by two independent angiographers who were unaware of MMP-9 results, and final agreement was $90 \%$, with discordances being resolved by consensus.

\section{Statistical analysis}

Comparisons between groups were done by t-test or Mann-Whitney $\mathrm{U}$ test (as indicated) for continuous variables and by Fisher's exact test for discrete variables. Correlation analyses were done by Pearson test or Spearman test, as indicated. Multivariable logistic regression analysis was applied to identify whether MMP-9 was independently associated with coronary no-reflow. A receiver operating characteristic (ROC) curve was used to acquire appropriate sensitivity and specificity of MMP-9 to identify the best threshold value for no flow. All statistical analyses were performed using SAS version 9.13 (SAS Institute Inc., Cary, NC, USA). Statistical significance was determined when $\mathrm{P}<0.05$.

\section{Results}

\section{Clinical characteristics of patients}

The 65 patients who underwent primary PCI on the IRA, 19 (29.3\%) developed no-reflow after the procedure. Table 1 shows a comparison of the baseline characteristics of the patients, and blood samples were drawn from a brachial vein in all patients before PCI.

\section{Angiographic and procedural characteristics}

Angiographic and procedural data are listed in Table 2. It was observed that the no-reflow group mainly consisted of patients with lesion length, reference luminal diameter, high thrombus score and lesion MMP-9 levels, which was determined from blood samples taken from lesions before balloon angioplasty. However, the presence of multivessel disease, IRA, target lesion locations and lesion types in subtotal occlusions showed no difference between the two groups $(\mathrm{P}>0.05)$.

\section{Regression analysis}

The slow flow of risk factors for $\mathrm{P}<0.2$ (including age, Systolic blood pressure, Triglycerides (mmol/L), Time to balloon (h), Lesion Length, Reference luminal diameter, Thrombus score, Lesion MMP-9 and Number of stents) included in the regression analysis, the observed indicator (Tables 3 and 4).

\section{ROC curve analysis}

The receiver operating characteristic curve revealed that a lesion MMP-9 change (ROC area: 0.740, 95\% CI: 0.607-0.872, $\mathrm{P}=0.002$ ) had predictive value for no reflow phenomenon (Figure 1). 
Citation: Guo F, Chai W, Liu M, Yu C, Liu W, et al. (2017) The Relationship between MMP-9 and Infarct Related Artery Reflow in Acute STEMI Patients. J Diabetes Metab 8: 749. doi:10.4172/2155-6156.1000749

Page 3 of 5

\begin{tabular}{|c|c|c|c|}
\hline & Reflow (n=46) & $\begin{array}{l}\text { No-reflow } \\
(n=19)\end{array}$ & $P$ value \\
\hline Age, year & $61.2 \pm 10.1$ & $65.7 \pm 11.0$ & 0.114 \\
\hline Male gender, \% & 28 & 10 & 0.54 \\
\hline $\mathrm{BMI}, \mathrm{kg} / \mathrm{m}$ & $24.7 \pm 2.2$ & $25.0 \pm 1.5$ & 0.573 \\
\hline Smoking, $\mathrm{n}$ & 26 & 12 & 0.621 \\
\hline Hypertension & 33 & 14 & 0.873 \\
\hline Diabetes mellitus, $\mathrm{n}$ & 19 & 11 & 0.222 \\
\hline Heart rate (bpm) & $74.0 \pm 10.8$ & $76.6 \pm 11.4$ & 0.399 \\
\hline $\begin{array}{l}\text { Systolic blood pressure } \\
(\mathrm{mmHg})\end{array}$ & $144.8 \pm 18.8$ & $134.1 \pm 23.8$ & 0.059 \\
\hline $\begin{array}{l}\text { Diastolic blood pressure } \\
(\mathrm{mmHg})\end{array}$ & $87.0 \pm 8.5$ & $84.2 \pm 7.9$ & 0.228 \\
\hline CK-MB peak (ng/ml) & $231.0 \pm 83.0$ & $249.7 \pm 82.8$ & 0.411 \\
\hline $\begin{array}{l}\text { Fasting blood glucose } \\
(\mathrm{mmol} / \mathrm{L})\end{array}$ & $9.0 \pm 3.1$ & $8.6 \pm 2.3$ & 0.628 \\
\hline Uric acid $(\mu$ & $301.5 \pm 63.8$ & $303.0 \pm 62.7$ & 0.929 \\
\hline Total cholesterol (mmol/L) & $5.44 \pm 1.25$ & $5.40 \pm 1.14$ & 0.892 \\
\hline HDL cholesterol (mmol/L) & $1.19 \pm 0.23$ & $1.13 \pm 0.23$ & 0.2 .72 \\
\hline LDL cholesterol (mmol/L) & $3.07 \pm 0.86$ & $3.22 \pm 0.79$ & 0.52 \\
\hline Triglycerides (mmol/L) & $1.62 \pm 0.62$ & $1.87 \pm 0.74$ & 0.164 \\
\hline MMP (pg/ml) & $25.8 \pm 6.9$ & $27.8 \pm 5.3$ & 0.447 \\
\hline \multicolumn{4}{|c|}{$\begin{array}{l}\text { BMI: body mass index; CK: Creatine kinase; MMP-9: matrix metalloproteinase- } 9 \\
\text { Note: * Statistically significant } \\
\text { Blood samples were drawn from a brachial vein in all patients before PCl }\end{array}$} \\
\hline
\end{tabular}

\begin{tabular}{|c|c|c|c|}
\hline & $\begin{array}{l}\text { Reflow } \\
(n=46)\end{array}$ & $\begin{array}{l}\text { No-reflow } \\
(n=19)\end{array}$ & $P$ value \\
\hline Time to balloon $(\mathrm{h})$ & $6.03 \pm 1.45$ & $6.76 \pm 1.49$ & 0.072 \\
\hline $\begin{array}{l}\text { Number of diseased vessels, } n \text {, } \\
\%\end{array}$ & $2.41 \pm 0.78$ & $2.58 \pm 0.77$ & 0.435 \\
\hline Culprit vessel, $\mathrm{n}$ & & & 0.761 \\
\hline LAD & 24 & 8 & \\
\hline LCX & 4 & 2 & \\
\hline RCA & 18 & 9 & \\
\hline Initial TIMI 0 flow, $\mathrm{n}$ & 36 & 17 & 0.289 \\
\hline Target lesion location, $\mathrm{n}$ & & & 0.512 \\
\hline Proximal & 25 & 9 & \\
\hline Mid & 19 & 10 & \\
\hline Distal & 2 & 0 & \\
\hline Lesion length (mm) & $29.0 \pm 13.2$ & $35.31 \pm 14.9$ & 0.01 \\
\hline Reference luminal diameter $(\mathrm{mm})$ & $3.97 \pm 0.46$ & $4.30 \pm 0.57$ & 0.016 \\
\hline Thrombus score $\geq 4$, n & 17 & 14 & $0.007^{*}$ \\
\hline Diver use, $\mathrm{n}, \%$ & 46,100 & 19,100 & 1 \\
\hline Lesion MMP-9 & $32.6 \pm 7.7$ & $42.1 \pm 7.1$ & $0.001^{*}$ \\
\hline Number of stents & $1.47 \pm 0.51$ & $1.68 \pm 0.58$ & 0.158 \\
\hline \multicolumn{4}{|c|}{$\begin{array}{l}\text { Note: * Statistically significant } \\
\text { Blood samples were drawn from the lesion before balloon angioplasty to } \\
\text { determine lesion MMP-9 }\end{array}$} \\
\hline
\end{tabular}

Table 1: General characteristics of patients.

\begin{tabular}{|l|l|l|l|l|}
\hline & OR & \multicolumn{2}{|l|}{$\begin{array}{l}\text { 95\% Wald } \\
\text { Confidence Limits }\end{array}$} & P value \\
\hline Age & 0.96 & 0.912 & 1.011 & 0.126 \\
\hline Time to balloon & 0.728 & 0.498 & 1.063 & 0.1 \\
\hline Systolic blood pressure & 1.021 & 0.994 & 1.049 & 0.122 \\
\hline Triglycerides & 0.597 & 0.272 & 1.31 & 0.199 \\
\hline Lesion length & 0.953 & 0.915 & 0.993 & $0.021^{*}$ \\
\hline Reference luminal diameter & 0.292 & 0.097 & 0.88 & $0.029^{*}$ \\
\hline Thrombus score & 0.229 & 0.074 & 0.707 & $0.010^{*}$ \\
\hline Stents & 0.385 & 0.137 & 1.081 & 0.07 \\
\hline Lesion MMP-9 & 0.892 & 0.825 & 0.964 & $0.004^{*}$ \\
\hline Note: * statistically significant & & & & \\
\hline
\end{tabular}

Table 2: Angiographic and procedural findings.

\begin{tabular}{|l|l|l|l|l|}
\hline & OR & \multicolumn{2}{|l|}{$\begin{array}{l}\text { 95\% Wald } \\
\text { Confidence Limits }\end{array}$} & P value \\
\hline Age & 0.969 & 0.901 & 1.042 & 0.392 \\
\hline Time to balloon & 0.777 & 0.435 & 1.387 & 0.394 \\
\hline Systolic blood pressure & 1.028 & 0.99 & 1.068 & 0.152 \\
\hline Triglycerides & 0.6 & 0.19 & 1.896 & 0.384 \\
\hline Lesion length & 0.929 & 0.845 & 1.021 & 0.126 \\
\hline Reference luminal diameter & 0.167 & 0.031 & 0.89 & $0.036^{*}$ \\
\hline Thrombus score & 0.085 & 0.014 & 0.506 & $0.007^{*}$ \\
\hline Stents & 1.3 & 0.132 & 12.793 & 0.822 \\
\hline Lesion MMP-9 & 0.881 & 0.791 & 0.981 & $0.021^{*}$ \\
\hline Note: * statistically significant & & & & \\
\hline
\end{tabular}

Table 4: Multivariable predictors of angiographic slow reflow.

Table 3: Univariate logistic regression analysis of slow reflow. 
Page 4 of 5

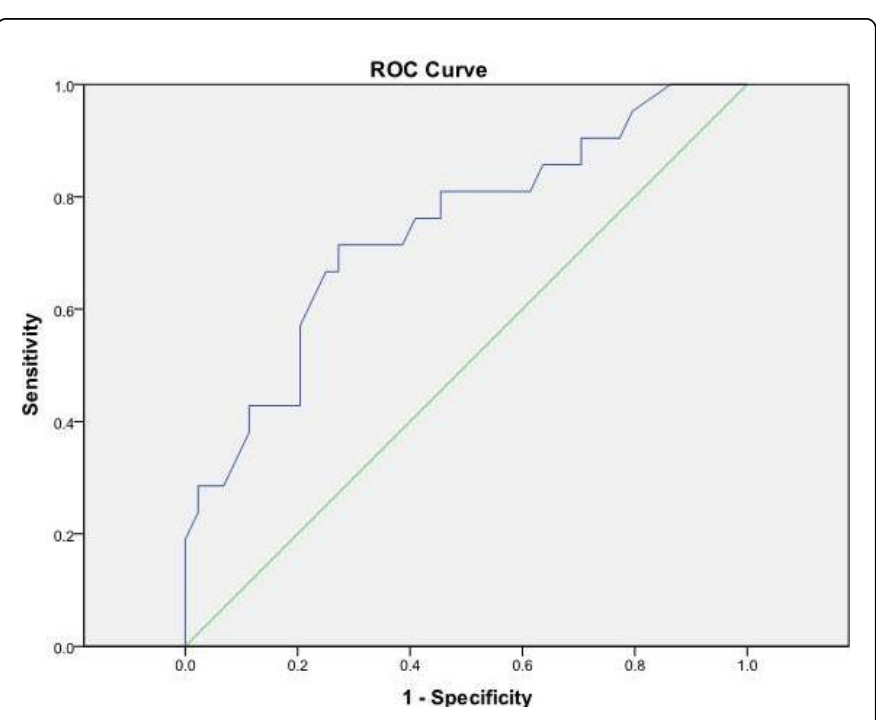

Figure 1: Receiver operator characteristic curve for lesion MMP-9 values with area under the curve(AUC) and 95\% confidence interval for AUC under the nonparametric assumption: approximate area under curve $=0.740$, Standard error $=0.067,95 \%$ Wald confidence limits $(0.607-0.872, \mathrm{P}=0.002)$.

\section{Discussion}

We found that the lesion site, rather than peripheral parts of the MMP-9 levels increased, affecting the coronary re-flow after stenting. Accurate diagnosis in patients with coronary heart disease, early risk stratification and effective treatment can significantly reduce the incidence of cardiovascular complications and mortality $[13,14]$. Recently, elective or primary PCI can significantly benefit to the patient with acute coronary syndrome [15-17]. Although the mortality of PCI perioperative is less than $3 \%$, and gradually reduced, but allcause mortality in patients with coronary heart disease is still high $[18,19]$. The emergence of drug-eluting stents significantly reduced the risk of stenosis of coronary artery and the ratio of the essential for revascularization of coronary heart disease, but no-reflow or slow reflow of opening the infracted related artery in the post-operative patients weakened the benefit of PCI [20,21].

We found that the level of MMP-9 in the lesion was higher than that in the peripheral coronary artery after PCI, which was closely related to TIMI blood flow after PCI, suggesting that activation of local inflammatory factors may be involved in the activity or slow reflow mechanism. PCI can lead to changes in the level of peripheral MMP-9, suggesting that the intervention itself may also stimulate the body to produce inflammatory response; and the level of lesion MMP-9, although subtle changes, we still believe that preoperative local inflammatory cytokines may be involved in postoperative slow reflow occurs.

Atherosclerosis is the basic process of coronary heart disease, inflammation plays an important role in this process. The process of atherosclerosis including monocytes and T-lymphocyte migration from the blood stream to the vessel wall, and lipoproteins penetrate and gather in the vessel wall by the support of adhesion molecules and growth regulating factors, and subsequently modified of lipoproteins stimulate leukocyte recruitment, bring about inflammation [22].
Recent studies have found that fragmentation can be induced inflammation associated with the damage by leukocyte recruited in the matrix tissue [23]. The non-specific collagen and elastic peptide fragments can also induce neutrophils, monocytes and fibroblasts to chemotactic response. Glycosaminogly can fragments can cause tissue damage, may be involved in regulating inflammation, hyaluronic acid fragments induce the expression of multiple inflammatory genes by macrophages and endothelial cells [24], suggest that it may has an important role in the process of regulate inflammation, and hyaluronic acid fragments removed from the injury tissue is required of chronic inflammation disappeared.

This study has also found that lesion MMP-9 was significantly higher than the outer periphery, suggesting their involvement in plaque rupture and acute occlusion. Some scholars have found that MMP-9 levels in vessel is higher than that in the coronary sinus and femoral arteries in the patients with acute myocardial infarction [25], so we believed that this increase was caused by plaque rupture rather than myocardial infarction.

Although this study was not able to measure the size of the plaque, we found that, after removal of the system level, MMP-9 also involved in the intervention slow reflow, suggesting that fragmentation of the plaque is still persistent inflammatory activity. In addition, IMMP-9 has been reported to have procoagulant activity [26], indicating that localized inflammatory response may also be a direct result of slow reflow.

In summary, it showed that local but not systemic MMP-9 was higher in patients with no reflow/slow reflow after PCI than in those with successful reperfusion. The results suggest that MMP-9 may be both involved and a prognostic marker of no reflow phenomenon.

\section{Conflicts of Interest}

All of the authors declare that they have no conflicts of interest regarding this paper.

\section{References}

1. Rodriguez D, Morrison CJ, Overall CM (2010) Matrix metalloproteinases: what do they not do? New substrates and biological roles identified by murine models and proteomics. Biochim Biophys Acta 1803: 39-54.

2. Silvello D, Narvaes LB, Albuquerque LC, Forgiarini LF, Meurer L, et al. (2014) Serum levels and polymorphisms of matrix metalloproteinases (MMPs) in carotid artery atherosclerosis: higher MMP-9 levels are associated with plaque vulnerability. Biomarkers. 19: 49-55.

3. Beaudeux JL, Giral P, Bruckert E, Foglietti MJ, Chapman MJ, et al. (2004) Matrix metalloproteinases, inflammation and atherosclerosis: therapeutic perspectives. Clin Chem Lab Med. 42: 121-131.

4. Song Z, Bai J, Zhang L, Sun X, Zhang M, et al. (2014) Effects of telmisartan on inflammation and fibrosis after acute myocardial infarction in rats. Zhonghua Yi Xue Za Zhi 94: 2628-2633.

5. Newby AC (2005) Dual role of matrix metalloproteinases (matrixins) in intimal thickening and atherosclerotic plaque rupture. Physiol Rev 85: $1-31$.

6. Hernandez-Anzaldo S, Brglez V, Hemmeryckx B, Leung D, Filep JG, et al. (2016) Novel Role for Matrix Metalloproteinase 9 in Modulation of Cholesterol Metabolism. J Am Heart Assoc 5: e004228.

7. Pinto AR, Godwin JW, Chandran A, Hersey L, Ilinykh A, et al. (2014) Age-related changes in tissue macrophages precede cardiac functional impairment. Aging (Albany NY) 6: 399-413. 
8. Xu S, Tang L, Mi Y, Jiang J, Zhu M, et al. (2013) Clinical significance of leukotriene b4 and extracellular matrix metalloproteinase inducer in acute coronary syndrome. Clin Invest Med 36: E282-E289.

9. Warren J, Mehran R, Yu J, Xu K, Bertrand ME, et al. (2015) Incidence and impact of totally occluded culprit coronary arteries in patients presenting with non-ST-segment elevation myocardial infarction. Am J Cardiol 115: 428-433.

10. Piccolo R, Niglio T, Spinelli L, Capuano E, Strisciuglio T, et al. (2014) Reperfusion correlates and clinical outcomes of right ventricular dysfunction in patients with inferior ST-segment elevation myocardial infarction undergoing percutaneous coronary intervention. Am J Cardiol 114: 243-249.

11. Celik T, Kaya MG, Akpek M, Yarlioglues M, Sarli B, et al. (2014) Does Serum Bilirubin level on admission predict TIMI flow grade and inhospital MACE in patients with STEMI undergoing primary PCI. Angiology 65: 198-204.

12. Karacsonyi J, Karatasakis A, Karmpaliotis D, Alaswad K, Yeh RW, et al. (2016) Effect of Previous Failure on Subsequent Procedural Outcomes of Chronic Total Occlusion Percutaneous Coronary Intervention (from a Contemporary Multicenter Registry). Am J Cardiol 117:1267-1271.

13. Wang X, Shi LZ (2015) Association of matrix metalloproteinase-9 $\mathrm{C} 1562 \mathrm{~T}$ polymorphism and coronary artery disease: a meta-analysis. J Zhejiang Univ Sci B 15: 256-263.

14. Greve SV, Blicher MK, Sehestedt T, Gram-Kampmann EM, Rasmussen S, et al. (2015) Effective risk stratification in patients with moderate cardiovascular risk using albuminuria and atherosclerotic plaques in the carotid arteries. J Hypertens 33: 1563-570.

15. Roed T, Kristoffersen US, Knudsen A, Wiinberg N, Lebech AM, et al. (2014) Increased prevalence of coronary artery disease risk markers in patients with chronic hepatitis C-a cross-sectional study. Vasc Health Risk Manag 10: 55-62.

16. Rigamonti F, Gencer B, Rey F, Chaara J, Tessitore E, et al. (2016) Prehospital alarm activation for STEMI patients undergoing primary percutaneous coronary intervention in the era of transradial procedures. Eur J Intern Med 35: 83-88.

17. Martinez-Sanchez C, Borrayo G, Carrillo J, Juarez U, Quintanilla J, et al. (2016) Clinical management and hospital outcomes of acute coronary syndrome patients in Mexico: The Third National Registry of Acute Coronary Syndromes (RENASICA III).Arch Cardiol Mex 86: 221-232.

18. Dharma S, Andriantoro H, Purnawan I, Dakota I, Basalamah F, et al (2016) Characteristics, treatment and in-hospital outcomes of patients with STEMI in a metropolitan area of a developing country: an initial report of the extended Jakarta Acute Coronary Syndrome registry. BMJ Open 6: e012193.

19. Jankowska-Polańska B, Uchmanowicz I, Dudek K, Łoboz-Grudzień K (2016) Sex differences in the quality of life of patients with acute coronary syndrome treated with percutaneous coronary intervention after a 3-year follow-up. Patient Prefer Adherence 10: 1279-1287.

20. Kim MC, Cho JY, Jeong HC, Lee KH, Park KH, et al. (2016) Long-Term Clinical Outcomes of Transient and Persistent No Reflow Phenomena following Percutaneous Coronary Intervention in Patients with Acute Myocardial Infarction. Korean Circ J 46: 490-498.

21. Wang L, Liu G, Liu J, Zheng M, Li L (2016) Effects of no-reflow phenomenon on ventricular systolic synchrony in patients with acute anterior myocardial infarction after percutaneous coronary intervention. Ther Clin Risk Manag 12: 1017-1022.

22. Silvestre-Roig C, de Winther MP, Weber C, Daemen MJ, Lutgens E, et al. (2014) Atherosclerotic plaque destabilization: mechanisms, models, and therapeutic strategies. Circ Res 114: 214-226.

23. Nakashiro S, Matoba T, Umezu R, Koga J, Tokutome M, et al. (2016) Pioglitazone-Incorporated Nanoparticles Prevent Plaque Destabilization and Rupture by Regulating Monocyte/Macrophage Differentiation in ApoE-/-Mice. Arterioscler Thromb Vasc Biol 36: 491-500.

24. Gibson CM, Schömig A (2004) Coronary and myocardial angiography: angiographic assessment of both epicardial and myocardial perfusion. Circulation 109: 3096-3105.

25. Kai H, Ikeda H, Yasukawa H, Kai M, Seki Y, et al. (1998) Peripheral blood levels of matrix metalloproteases-2 and -9 are elevated in patients with acute coronary syndromes. J Am Coll Cardiol 32: 368-372.

26. Nascimento-Silva V, Rodrigues da Silva G, Moraes JA, Cyrino FZ, Seabra $\mathrm{SH}$, et al. (2012) A pro-inflammatory profile of endothelial cell in Lonomia obliqua envenomation. Toxicon 60: 50-60. 